\title{
TABULATION OF THIN PLATE SPLINES ON A VERY FINE TWO-DIMENSIONAL GRID
}

\author{
by \\ M.J.D. Powell \\ Department of Applied Mathematics and Theoretical Physics, \\ University of Cambridge, England
}

No. 67

February, 1992

Abstract: A thin plate spline approximation has the form

$$
s(x)=\sum_{j=1}^{n} \lambda_{j}\left\|x-x_{j}\right\|_{2}^{2} \log \left\|x-x_{j}\right\|_{2}+p(x), \quad x \in \mathcal{R}^{2},
$$

where $\left\{\lambda_{j} \in \mathcal{R}: j=1,2, \ldots, n\right\}$ and $\left\{x_{j} \in \mathcal{R}^{2}: j=1,2, \ldots, n\right\}$ are parameters and where $p$ is a linear polynomial. There exist several applications that require $s$ to be tabulated at all the lattice points of a very fine square grid. For example, $10^{8}$ grid points and $n=500$ can occur, and then the direct evaluation of $s$ at every grid point would be impracticable. Fortunately each thin plate spline term is smooth away from its centre $x_{j}$, so it is possible to apply a scheme that subtabulates by finite differences provided that special attention is given to those terms whose centres are close to the current $x$. Thus the total work is bounded by a small constant multiple of the number of grid points plus a constant multiple of $n \epsilon^{-1 / 3}|\log h|$, where $\epsilon$ is a given tolerance on the calculated values of $s(x)$ and where $h$ is the mesh size of the fine grid. We will find that the exponent $-1 / 3$ is due to the order of the differences that are employed. An algorithm for this calculation is described and discussed and some numerical results are presented. The errors of the subtabulation procedures are studied in an appendix. 


\section{Introduction}

When a time-dependent system is under observation, one may wish to compare pictures of the system that are taken at regular intervals. Examples include monitoring the possible growth of a tumour in a hospital patient and observing the silting-up of a shipping channel. These two applications are mentioned because in both cases two kinds of differences may occur between one picture and the next. Of course one kind of change is due to the physical property that is being explored. On the other hand, some ambient properties of the picture may alter too, such as the amount of air in the patient's lungs and the state of the tide in the shipping lane. Other differences in the ambient conditions can include the position of the patient and of the vessel that is collecting data, perhaps by means of side-scan sonar measurements.

Therefore techniques that allow for the changes in the ambient conditions are needed. They depend on known relations between one picture and the next. The bone structure of the patient, for example, can provide suitable information, as can the positions of buoys, wrecks and rocks in the shipping channel. We address the case where such information can be identified precisely in the sequence of pictures that is being compared. Specifically, we let $\left\{\left(s_{j}, t_{j}\right): j=1,2, \ldots, n\right\}$ be the coordinates of points in one picture that are known to correspond to the points $\left\{\left(x_{j}, y_{j}\right): j=1,2, \ldots, n\right\}$ in another picture. Then we employ a transformation from the $(x, y)$ picture to the $(s, t)$ picture that satisfies the interpolation equations

$$
s\left(x_{j}, y_{j}\right)=s_{j} \quad \text { and } t\left(x_{j}, y_{j}\right)=t_{j}, \quad j=1,2, \ldots, n .
$$

Two questions have arisen. One is the choice of the mapping functions $s$ and $t$, each being from $\mathcal{R}^{2}$ to $\mathcal{R}$, and the other is the purpose of these functions. Of course $s$ and $t$ must have the ability to interpolate data in general position. Therefore, as in Barrodale, Berkley and Skea (1992), we assume that they are "thin plate splines", which means that they have the form

$$
\left.\begin{array}{l}
s(x, y)=\sum_{j=1}^{n} \lambda_{j}\left[\left(x-x_{j}\right)^{2}+\left(y-y_{j}\right)^{2}\right] \log \left[\left(x-x_{j}\right)^{2}+\left(y-y_{j}\right)^{2}\right]^{1 / 2}+a x+b y+c \\
t(x, y)=\sum_{j=1}^{n} \mu_{j}\left[\left(x-x_{j}\right)^{2}+\left(y-y_{j}\right)^{2}\right] \log \left[\left(x-x_{j}\right)^{2}+\left(y-y_{j}\right)^{2}\right]^{1 / 2}+d x+e y+f
\end{array}\right],
$$

where $\left\{\lambda_{j}: j=1,2, \ldots, n\right\}, a, b, c,\left\{\mu_{j}: j=1,2, \ldots, n\right\}, d, e$ and $f$ are parameters that are chosen to satisfy the interpolation equations (1.1). Usually the six remaining degrees of freedom are fixed by the conditions

$$
\sum_{j=1}^{n} \lambda_{j}=\sum_{j=1}^{n} \lambda_{j} x_{j}=\sum_{j=1}^{n} \lambda_{j} y_{j}=\sum_{j=1}^{n} \mu_{j}=\sum_{j=1}^{n} \mu_{j} x_{j}=\sum_{j=1}^{n} \mu_{j} y_{j}=0,
$$

because then the interpolants are solutions to variational problems that minimize second derivative norms of $s$ and $t$ (Duchon, 1977). Further, the variational 
principle ensures that the parameters are well-defined, provided that the points $\left\{\left(x_{j}, y_{j}\right): j=1,2, \ldots, n\right\}$ are distinct and not collinear. Our work does not depend on the constraints (1.3).

Having picked the transformation (1.2), we apply it to a discretization of the whole $(x, y)$ picture. The resultant image in $(s, t)$ space becomes suitable for comparison with the $(s, t)$ picture, because the mapping functions provide exact agreement between the interpolation points. The construction of the image in $(s, t)$ space may require the functions $(1.2)$ to be calculated for very many values of $(x, y)$, perhaps as many as $10^{8}$, while $n=200$ is a typical value of $n$. Our purpose is to show that, by taking advantage of the smoothness properties of thin plate splines, the amount of computation to perform this task can be much less than the total time of direct evaluation. We assume that the values $\{s(\ell h, m h)$ : $0 \leq \ell, m \leq M\}$ are required, where $M$ is a large integer and $h=1 / M$, and that it is sufficient to approximate these values to about 6-9 decimal places of accuracy. We make no further reference to the second line of expression (1.2), because $t$ can be treated in the same way as $s$.

Ian Barrodale suggested that I study this problem because he knew of my interest in radial basis function methods. The first application that he mentioned was a comparison of satellite pictures of the forests of British Columbia. Here very small values of $h$ can occur because the resolution of the pictures is so fine that the positions of road junctions and confluences of rivers are available as interpolation points. I provided Barrodale Computing Services with a Fortran package that performs the tabulation. We are going to consider the method that is used.

We let the initial value of $h$ be $2^{k}$ times its required final value for some integer $k$, which is chosen so that, if the number of points of the initial grid is between about $20 \times 20$ and $40 \times 40$, then this grid covers the $(s, t)$ picture with enough overlap to allow for some edge effects that will be explained later. The function $s(x, y)$ is calculated from formula (1.2) at the mesh points of the initial grid, except for a modification that will also be explained later, that is invoked when $(x, y)$ is within a certain distance of at least one of the interpolation points. Then an iterative procedure is employed $k$ times, where each iteration halves the value of $h$ by applying the method that is described in Sections 2 and 3 . Here the old and the new mesh sizes are $2 h$ and $h$ respectively. When $\ell$ and $m$ are odd integers and when the point $(\ell h, m h)$ is sufficiently far from all the interpolation points, then we approximate $s(\ell h, m h)$ by a linear combination of the 16 function values of the coarser grid that are contained in the square $[(\ell-3) h,(\ell+3) h] \times[(m-$ $3) h,(m+3) h]$, the coefficients of the linear combination being chosen so that the error of the approximation is of magnitude $\mathcal{O}\left(h^{6}\right)$. Another procedure provides $\mathcal{O}\left(h^{6}\right)$ estimates of $s(\ell h, m h)$ when $\ell+m$ is odd. These techniques are given in Section 2. They provide approximations of sufficient accuracy when the point $(\ell h, m h)$ of the finer mesh satisfies the conditions

$$
\max \left[\left|\ell h-x_{j}\right|,\left|m h-y_{j}\right|\right] \geq \rho h, \quad j=1,2, \ldots, n,
$$


where $\rho$ is a constant that depends on the required precision, a typical value being $\rho=15$. Otherwise some modifications to the calculation of $s(\ell h, m h)$ are necessary to provide sufficient accuracy. They are the subject of Section 3.

Condition (1.4) implies an upper bound on the total number of explicit evaluations of thin plate spline terms when the current mesh size is halved by the procedure of Sections 2 and 3. We will find that this number is of magnitude $\left[3 \rho^{2}+\mathcal{O}(\rho)\right] n$. Further, each new value of $s(\ell h, m h)$ requires a simple linear combination of at most 16 old values to be formed, whatever the number of terms in the sums of expression (1.2). Further, for each $j$, the Section 3 procedure includes some corrections at the boundary of the $j$-th constraint (1.4), the work of this task being at most a constant times $\rho$ for every $j$. Thus the computational effort of halving the mesh size is bounded above by a small multiple of the number of new grid points plus a large multiple of $n$. Since the number of halvings is $k \approx\left|\log _{2} h\right|$, where $h$ is now the final mesh size, it follows that the total work of our procedure is of magnitude $\mathcal{O}\left(h^{-2}+n\left|\log _{2} h\right|\right)$, which usually provides very substantial gains over the direct use of formula (1.2) when $h$ is tiny, because the number of operations of the direct approach is of magnitude $\mathcal{O}\left(h^{-2} n\right)$. Some numerical results illustrate this important point in Section 4, and they are followed by a brief discussion of the given algorithm.

The choice of $\rho$ for condition (1.4) depends on the accuracy of the subtabulation schemes of Section 2. Indeed, a recommended value of $\rho$ is derived from the $\mathcal{O}\left(h^{6}\right)$ terms of the subtabulation errors. We should ask, however, whether higher order terms can invalidate these $\mathcal{O}\left(h^{6}\right)$ error estimates for typical values of $h$. This question is addressed in an appendix. Fortunately we find that the recommended choice is suitable. Further, the analysis provides some explicit formulae for derivatives and Taylor series expansions of thin plate splines.

\section{The subtabulation procedures}

This section addresses the problem of estimating a function $f$ on a square grid of mesh size $h$, when $f(\ell h, m h)$ is available for even values of the integers $\ell$ and $m$. Further, we assume that $f$ satisfies the biharmonic equation

$$
\frac{\partial^{4} f(x, y)}{\partial x^{4}}+2 \frac{\partial^{4} f(x, y)}{\partial x^{2} \partial y^{2}}+\frac{\partial^{4} f(x, y)}{\partial y^{4}}=0,
$$

because thin plate splines have this property away from their interpolation points. It is helpful to regard $f$ as a substitute for $s$ that does not have any singularities, because we are going to derive local error estimates from Taylor series expansions. First we pick an approximation to $f(\ell h, m h)$ when both $\ell$ and $m$ are odd integers.

This approximation is shown in Figure 1. One should relate the figure to a $7 \times 7$ grid of mesh size $h$, where 16 of the points belong to a grid of size $2 h$ and where $(\ell h, m h)$ is the central point of the picture. Further, the numbers in the 


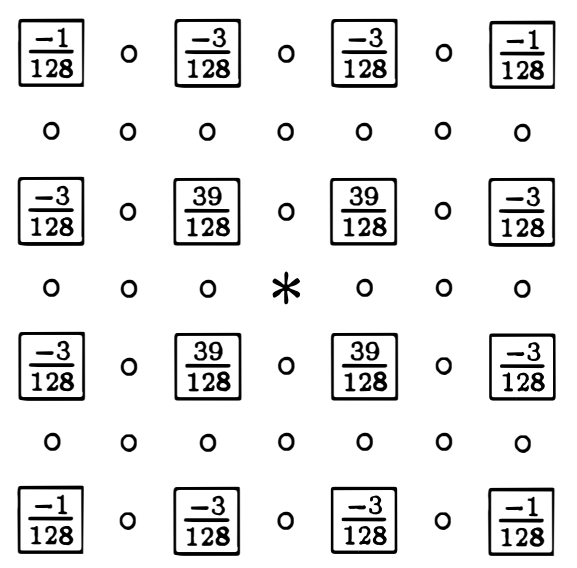

Figure 1

The 16-point subtabulation stencil

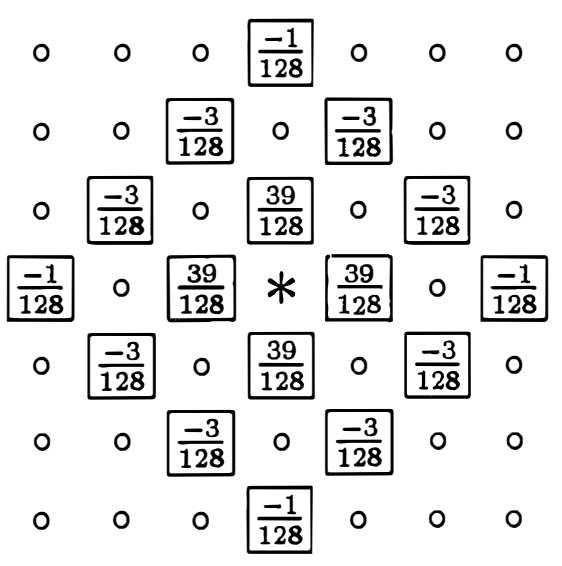

Figure 2

The rotated 16-point stencil

boxes are the factors that multiply the given values of $f$ in the approximation to $f(\ell h, m h)$. Therefore the figure depicts the estimate

$$
\begin{aligned}
f(\ell h, m h) \approx \sum_{ \pm, \pm} & {\left[\frac{39}{128} f(\ell h \pm h, m h \pm h)-\frac{3}{12} \overline{8} f(\ell h \pm 3 h, m h \pm h)\right.} \\
& \left.-\frac{3}{128} f(\ell h \pm h, m h \pm 3 h)-\frac{1}{12} \overline{8} f(\ell h \pm 3 h, m h \pm 3 h)\right] .
\end{aligned}
$$

The numbers in Figure 1 were chosen in the following way to maximize the order of the error of this approximation to $f(\ell h, m h)$.

Let the factors $\frac{39}{128}, \frac{-3}{128}$ and $\frac{-1}{128}$ in formula $(2.2)$ be called $\alpha, \beta$ and $\gamma$. The symmetry implies that the error is zero when $f$ is any quadratic polynomial if and only if it is zero when $f(x, y)=1$ and when $f(x, y)=x^{2}$. Therefore we require the equations

$$
4 \alpha+8 \beta+4 \gamma=1 \text { and } \alpha+10 \beta+9 \gamma=0
$$

to be satisfied. Further, by identifying $(\ell h, m h)$ with the origin in the Taylor series expansion

$$
f(x, y)=\sum_{j=0}^{\infty} \sum_{k=0}^{\infty} \frac{x^{j}}{j !} \frac{y^{k}}{k !} \frac{\partial^{j+k} f(0,0)}{\partial x^{j} \partial y^{k}},
$$

we deduce that the fourth order part of the error is the expression

$$
\begin{aligned}
& 4 \alpha\left[\frac{h^{4}}{4 !} \frac{\partial^{4} f(0,0)}{\partial x^{4}}+\frac{h^{2}}{2 !} \frac{h^{2}}{2 !} \frac{\partial^{4} f(0,0)}{\partial x^{2} \partial y^{2}}+\frac{h^{4}}{4 !} \frac{\partial^{4} f(0,0)}{\partial y^{4}}\right] \\
& +4 \beta\left[\frac{(3 h)^{4}}{4 !} \frac{\partial^{4} f(0,0)}{\partial x^{4}}+\frac{(3 h)^{2}}{2 !} \frac{h^{2}}{2 !} \frac{\partial^{4} f(0,0)}{\partial x^{2} \partial y^{2}}+\frac{h^{4}}{4 !} \frac{\partial^{4} f(0,0)}{\partial y^{4}} .\right. \\
& \left.+\frac{h^{4}}{4 !} \frac{\partial^{4} f(0,0)}{\partial x^{4}}+\frac{h^{2}}{2 !} \frac{(3 h)^{2}}{2 !} \frac{\partial^{4} f(0,0)}{\partial x^{2} \partial y^{2}}+\frac{(3 h)^{4}}{4 !} \frac{\partial^{4} f(0,0)}{\partial y^{4}}\right]
\end{aligned}
$$




$$
\begin{gathered}
+4 \gamma\left[\frac{(3 h)^{4} \partial^{4} f(0,0)+\frac{(3 h)^{2}(3 h)^{2} \partial^{4} f(0,0)}{2 !} \partial x^{4}+\frac{(3 h)^{4} \partial^{4} f(0,0)}{2 !} 2 !}{\partial x^{2} \partial y^{2}}\right] \\
=\frac{1}{6} h^{4}\left[(\alpha+82 \beta+81 \gamma)\left(\frac{\partial^{4} f(0,0)}{\partial x^{4}}+\frac{\partial^{4} f(0,0)}{\partial y^{4}}\right)\right. \\
\left.+(6 \alpha+108 \beta+486 \gamma) \frac{\partial^{4} f(0,0)}{\partial x^{2} \partial y^{2}}\right] .
\end{gathered}
$$

Therefore, in view of equation (2.1), this expression is zero if we force the relation

$$
\alpha+82 \beta+81 \gamma=3 \alpha+54 \beta+243 \gamma .
$$

The conditions (2.3) and (2.6) define the coefficients that are shown in Figure 1 and formula (2.2).

Therefore, if the error of the approximation (2.2) is expanded in powers of $h$, the leading nonzero term is of magnitude $\mathcal{O}\left(h^{6}\right)$. Specifically, the analogue of expression (2.5) gives the term

$$
\begin{aligned}
& \frac{1}{180} h^{6}\left[(\alpha+730 \beta+729 \gamma)\left(\frac{\partial^{6} f(0,0)}{\partial x^{6}}+\frac{\partial^{6} f(0,0)}{\partial y^{6}}\right)\right. \\
& \left.+(15 \alpha+1350 \beta+10935 \gamma)\left(\frac{\partial^{6} f(0,0)}{\partial x^{4} \partial y^{2}}+\frac{\partial^{6} f(0,0)}{\partial x^{2} \partial y^{4}}\right)\right] \\
& =-\frac{1}{8} h^{6}\left(\frac{\partial^{6} f(0,0)}{\partial x^{6}}+5 \frac{\partial^{6} f(0,0)}{\partial x^{4} \partial y^{2}}+5 \frac{\partial^{6} f(0,0)}{\partial x^{2} \partial y^{4}}+\frac{\partial^{6} f(0,0)}{\partial y^{6}}\right) .
\end{aligned}
$$

Invoking the identity

$$
\frac{\partial^{6} f(0,0)}{\partial x^{6}}+3 \frac{\partial^{6} f(0,0)}{\partial x^{4} \partial y^{2}}+3 \frac{\partial^{6} f(0,0)}{\partial x^{2} \partial y^{4}}+\frac{\partial^{6} f(0,0)}{\partial y^{6}}=0
$$

which is obtained by applying the Laplacian operator to equation (2.1), we write expression (2.7) in the form

$$
\frac{1}{12} h^{6}\left(\frac{\partial^{6} f(0,0)}{\partial x^{6}}+\frac{\partial^{6} f(0,0)}{\partial y^{6}}\right) \text {. }
$$

It follows that, if $f$ is any smooth function that satisfies the biharmonic equation, then the approximation (2.2) has the error

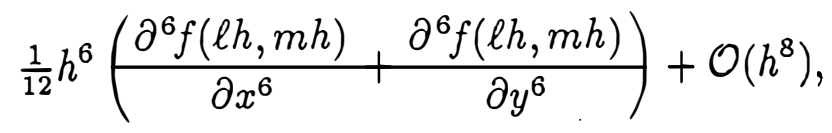

which will be used in the next section to pick the constant $\rho$ that occurs in condition (1.4). 


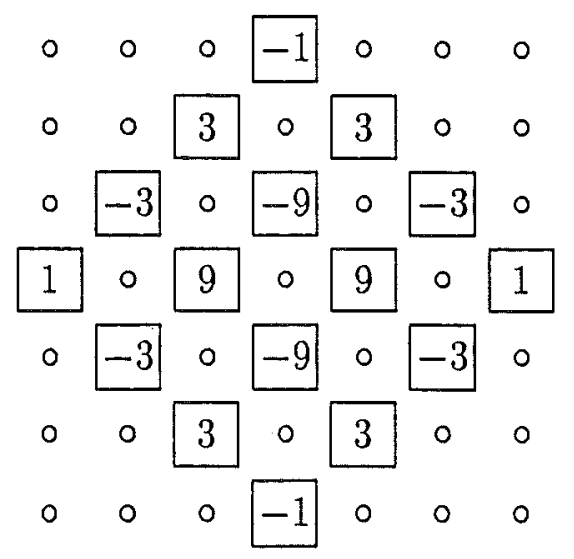

Figure 3

An $\mathcal{O}\left(h^{6}\right)$ functional

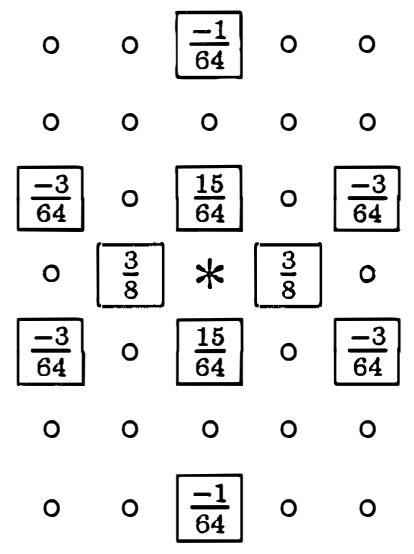

Figure 4

A 10-point subtabulation stencil

We also require a procedure that provides a sufficiently accurate approximation to $f(\ell h, m h)$ when $\ell+m$ is odd. We assume now that $f(\ell h, m h)$ is available whenever $\ell+m$ is even, the values when $\ell$ and $m$ are both even being data and the values when $\ell$ and $m$ are both odd having been generated by formula (2.2). Therefore the stencil in Figure 2 is suitable, which is obtained by rotating the stencil of Figure 1 through the angle $\pi / 4$ and scaling it by the factor $2^{-1 / 2}$. There are advantages in using a narrower stencil, however. Therefore we derive the stencil in Figure 4 by adding 1/128 times the stencil in Figure 3 to the one in Figure 2, which gives the algebraic formula

$$
\begin{gathered}
f(\ell h, m h) \approx \sum_{ \pm}\left[\frac{3}{8} f(\ell h \pm h, m h)+\frac{15}{64} f(\ell h, m h \pm h)-\frac{1}{64} f(\ell h, m h \pm 3 h)\right] \\
-\frac{3}{64} \sum_{ \pm, \pm} f(\ell h \pm 2 h, m h \pm h) .
\end{gathered}
$$

The error of this approximation is also of magnitude $\mathcal{O}\left(h^{6}\right)$, because it can be verified that Figure 3 depicts a linear combination of function values that vanishes for all quintic polynomials, which is easy to see if Figure 3 is rotated through the angle $\pi / 4$. Further, by rotating Figure 4 through $\pi / 2$, we find the $\mathcal{O}\left(h^{6}\right)$ approximation

$$
\begin{gathered}
f(\ell h, m h) \approx \sum_{ \pm}\left[\frac{3}{8} f(\ell h, m h \pm h)+\frac{15}{64} f(\ell h \pm h, m h)-\frac{1}{6 \overline{4}} f(\ell h \pm 3 h, m h)\right] \\
-\frac{3}{64} \sum_{ \pm, \pm} f(\ell h \pm h, m h \pm 2 h) .
\end{gathered}
$$

The Fortran software applies formula (2.2) when $\ell$ and $m$ are both odd, formula (2.11) when $\ell$ is even and $m$ is odd, and formula (2.12) when $\ell$ is odd and $m$ is 


\begin{tabular}{|c|c|c|c|c|c|c|c|c|}
\hline$\oplus$ & $\oplus$ & $\oplus$ & & $\oplus$ & $\oplus$ & $\oplus$ & & $\oplus$. \\
\hline \multirow[t]{2}{*}{$\oplus$} & $\oplus$ & $\oplus$ & & $\oplus$ & $\oplus$ & $\oplus$ & & $\oplus \cdots$ \\
\hline & $*$ & $\Leftrightarrow$ & $*$ & $\Leftrightarrow *$ & $\Leftrightarrow *$ & $\Leftrightarrow$ & $*$ & $\Leftrightarrow \ldots$ \\
\hline \multirow[t]{2}{*}{$\oplus$} & $\oplus \mathbb{\Downarrow}$ & $\oplus$ & $\Uparrow$ & $\oplus \mathbb{\Downarrow}$ & $\oplus \Uparrow$ & $\oplus$ & $\Uparrow$ & $\oplus \cdots$ \\
\hline & $*$ & $\Leftrightarrow$ & $*$ & $\Leftrightarrow *$ & $\Leftrightarrow *$ & $\Leftrightarrow$ & $*$ & $\Leftrightarrow$. \\
\hline \multirow[t]{2}{*}{$(1$} & $\oplus \mathbb{\Downarrow}$ & $\oplus$ & $\Uparrow$ & $\varphi-\Uparrow$ & $-\Uparrow$ & $\infty$ & $-\uparrow-$ & \\
\hline & $*$ & $\Leftrightarrow$ & $*$ & $\stackrel{\prime}{\Leftrightarrow} *$ & $\Leftrightarrow *$ & $\Leftrightarrow$ & $*$ & \\
\hline \multirow[t]{2}{*}{$\oplus$} & $\oplus \mathbb{\Downarrow}$ & $\oplus$ & $\Uparrow$ & $\oint \mathbb{I}$ & $\oplus \Uparrow$ & $\oplus$ & $\Uparrow$ & $\oplus$ \\
\hline & $*$ & $\Leftrightarrow$ & $*$ & $\stackrel{1}{\Leftrightarrow} *$ & $\Leftrightarrow *$ & $\Leftrightarrow$ & $*$ & $\Leftrightarrow$ \\
\hline & $\oplus \mathbb{\Downarrow}$ & $\oplus$ & $\Uparrow$ & $\phi \mathbb{1}$ & $\oplus \mathbb{\Downarrow}$ & $\oplus$ & $\Uparrow$ & $\oplus$ \\
\hline & & & & $! \quad \vdots$ & $\vdots \quad \vdots$ & $\vdots$ & $\vdots$ & $\vdots$ \\
\hline
\end{tabular}

Subtabulation at a corner of the grid

even. Thus most of the function values on the right hand sides of expressions (2.11) and (2.12) are data from previous calculations, rather than estimates that have been generated by equation (2.2) for the current $h$. This strategy is particularly convenient at the grid boundaries. Indeed, Figure 5 depicts the top left hand corner of a large square grid, where " $\oplus$ " denotes the points $(\ell h, m h)$ at which both $\ell$ and $m$ are even. Therefore the asterisks indicate the points $(\ell h, m h)$ for which all the function values on the right hand side of formula (2.2) are available. Having calculated these function values, formulae (2.11) and (2.12) are applicable and provide estimates of $f(\ell h, m h)$ at the points that are indicated by " $\Leftrightarrow$ " and "1)" respectively.

It is important to note that, if the subtabulation procedure is applied recursively to halve the grid size many times, then the given formulae can provide estimates of all the required function values to the right of and below the solid lines of Figure 5. Indeed, the figure shows that the finer grid inherits from the coarser grid three complete lines of function values at mesh points that are outside the solid lines, and of course this property can be passed on to all subsequent calculations that halve the mesh size. Therefore, because we are supposing that the final grid should cover the square $[0,1] \times[0,1]$, we let the coverage of each intermediate grid of mesh size $2 h$ be exactly the square $\left[-6 h, M_{h} h\right] \times\left[-6 h, M_{h} h\right]$, where $M_{h}$ is the least even integer that satisfies the inequality $M_{h} h \geq 1+6 h$. This construction provides the overlap that is mentioned in Section 1. 


\section{Removal of the singularities}

We modify the obvious choice $f=s$ in the subtabulation formulae (2.2), (2.11) and (2.12) when the integers $\ell$ and $m$ fail to satisfy all the conditions (1.4), where the constant $\rho$ will be specified in this section. In order to describe the procedure, we let $\mathcal{N}_{j}(h)$ be the neighbourhood

$$
\mathcal{N}_{j}(h)=\left\{(x, y): \max \left[\left|x-x_{j}\right|,\left|y-y_{j}\right|\right]<\rho h\right\} \subset \mathcal{R}^{2}
$$

of $\left(x_{j}, y_{j}\right)$, we let $\mathcal{J}_{h}(x, y)$ be the set

$$
\mathcal{J}_{h}(x, y)=\left\{j:(x, y) \notin \mathcal{N}_{j}(h)\right\} \cap\{1,2, \ldots, n\},
$$

and we let $s_{h}$ be the function that takes the values

$$
s_{h}(x, y)=\sum_{j \in \mathcal{J}_{h}(x, y)} \lambda_{j}\left[\left(x-x_{j}\right)^{2}+\left(y-y_{j}\right)^{2}\right] \log \left[\left(x-x_{j}\right)^{2}+\left(y-y_{j}\right)^{2}\right]^{1 / 2}+a x+b y+c .
$$

In other words, $s_{h}(x, y)$ is the same as the first line of expression (1.2), except that we have deleted the contributions to $s(x, y)$ from any interpolation points whose $\infty$-norm distance to $(x, y)$ is less than $\rho h$. Thus $s_{h}$ usually has discontinuities at the boundaries of the neighbourhoods $\left\{\mathcal{N}_{j}(h): j=1,2, \ldots, n\right\}$. When the Fortran implementation of the procedure of Section 2 applies the subtabulation formulae that reduce the mesh size from $2 h$ to $h$, it works with values of $s_{h}$ at the grid points instead of with values of $s$. Therefore it is possible to let $\rho$ be so large that the errors that arise from each singularity of the thin plate spline are tolerable. Then the following procedure includes techniques that allow for the discontinuities in $s_{h}$ and for the dependence of $s_{h}$ on $h$.

Given the values of $s_{h}(\ell h, m h)$ for all even values of $\ell$ and $m$, where the grid of mesh size $2 h$ covers the square $\left[-6 h, M_{h} h\right] \times\left[-6 h, M_{h} h\right]$ that is mentioned at the end of Section 2, the software applies the method of that section to generate function values on the grid of mesh size $h$ that covers the square $\left[-3 h, M_{h / 2} \frac{1}{2} h\right] \times$ $\left[-3 h, M_{h / 2} \frac{1}{2} h\right]$. Then the new function values are corrected where necessary so that they all become values of $s_{h}$ to within the $\mathcal{O}\left(h^{6}\right)$ accuracy of the subtabulation formulae. Indeed, for every $j$, we have to revise the function value at $(\ell h, m h)$ if $\ell$ and $m$ are not both even and if the $\infty$-norm distance from $(\ell h, m h)$ to the boundary of $\mathcal{N}_{j}(h)$ is less than $3 h$. This calculation is done explicitly and is often the most expensive part of the entire computation. Thus adequate estimates of $s_{h}$ are generated on the finer grid. Then they are overwritten by values of $s_{h / 2}$ by adding to expression (3.3) the contributions from the integers $j$ that are in the set $\mathcal{J}_{h / 2}(x, y) \backslash \mathcal{J}_{h}(x, y)$. Here, instead of taking the view that $(x, y)$ ranges over the points of the finer grid, one should treat the values of $j$ in sequence, adding in all the differences between $s_{h / 2}$ and $s_{h}$ for each $j$ before turning to a new value of $j$. Indeed, the total work of the latter approach is $\mathcal{O}\left(n \rho^{2}\right)$ operations for every $h$, 
but an $\mathcal{O}\left(h^{-2}\right)$ overhead would occur if one considered each point of the finer grid individually. The number of thin plate spline terms that are evaluated during these operations for each $h$ is of magnitude $\left[3 \rho^{2}+\mathcal{O}(\rho)\right] n$, as mentioned in Section 1 . Further, when $h$ reaches its final value, the numbers $\left\{s_{h / 2}(\ell h, m h): 0 \leq \ell, m \leq M\right\}$ have to be replaced by values of $s$. Therefore the calculation is completed by a cycle through the neighbourhoods $\left\{\mathcal{N}_{j}\left(\frac{1}{2} h\right): j=1,2, \ldots, n\right\}$ that adds the term

$$
\lambda_{j}\left[\left(\ell h-x_{j}\right)^{2}+\left(m h-y_{j}\right)^{2}\right] \log \left[\left(\ell h-x_{j}\right)^{2}+\left(m h-y_{j}\right)^{2}\right]^{1 / 2}
$$

to $s_{h / 2}(\ell h, m h)$ for every grid point $(\ell h, m h)$ that is in $\mathcal{N}_{j}\left(\frac{1}{2} h\right)$, so there are about another $n \rho^{2}$ evaluations of thin plate spline terms.

We now turn to the choice of $\rho$, letting $h$ be the generic mesh size of a cycle through the calculations of Section 2. When $f$ is the function $s_{h}$ in the subtabulation formula (2.2), it follows from expressions (2.10) and (3.3) that the estimate of $s_{h}(\ell h, m h)$ has the truncation error

$$
\frac{1}{12} h^{6} \sum_{j \in \mathcal{J}_{h}(\ell h, m h)} \lambda_{j}\left(\frac{\partial^{6} \phi\left(\ell h-x_{j}, m h-y_{j}\right)}{\partial x^{6}}+\frac{\partial^{6} \phi\left(\ell h-x_{j}, m h-y_{j}\right)}{\partial y^{6}}\right)+\mathcal{O}\left(h^{8}\right),
$$

where $\phi$ is the function

$$
\phi(x, y)=\left(x^{2}+y^{2}\right) \log \left(x^{2}+y^{2}\right)^{1 / 2}, \quad(x, y) \in \mathcal{R}^{2},
$$

and where we are ignoring the consequences of discontinuities in $s_{h}$, because we have noted already that they are treated explicitly. Therefore $\rho$ depends on sixth derivatives of $\phi$. We calculate the values

$$
\left.\begin{array}{l}
\partial \phi(x, y) / \partial x=x \log \left(x^{2}+y^{2}\right)+x \\
\partial^{2} \phi(x, y) / \partial x^{2}=\log \left(x^{2}+y^{2}\right)+1+2 x^{2} /\left(x^{2}+y^{2}\right) \\
\partial^{3} \phi(x, y) / \partial x^{3}=\left(2 x^{3}+6 x y^{2}\right) /\left(x^{2}+y^{2}\right)^{2} \\
\partial^{4} \phi(x, y) / \partial x^{4}=\left(-2 x^{4}-12 x^{2} y^{2}+6 y^{4}\right) /\left(x^{2}+y^{2}\right)^{3} \\
\partial^{5} \phi(x, y) / \partial x^{5}=\left(4 x^{5}+40 x^{3} y^{2}-60 x y^{4}\right) /\left(x^{2}+y^{2}\right)^{4} \\
\partial^{6} \phi(x, y) / \partial x^{6}=\left(-12 x^{6}-180 x^{4} y^{2}+540 x^{2} y^{4}-60 y^{6}\right) /\left(x^{2}+y^{2}\right)^{5}
\end{array}\right\}
$$

and then symmetry provides the formula

$$
\frac{\partial^{6} \phi(x, y)}{\partial x^{6}}+\frac{\partial^{6} \phi(x, y)}{\partial y^{6}}=\left(-72 x^{4}+432 x^{2} y^{2}-72 y^{4}\right) /\left(x^{2}+y^{2}\right)^{4} \text {. }
$$

Further, by combining the elementary identities

$$
x^{4}-6 x^{2} y^{2}+y^{4}=\left(x^{2}+y^{2}\right)^{2}-8 x^{2} y^{2}=2\left(x^{2}-y^{2}\right)^{2}-\left(x^{2}+y^{2}\right)^{2}
$$

with equation (3.8), we obtain the bound

$$
\left|\frac{\partial^{6} \phi(x, y)}{\partial x^{6}}+\frac{\partial^{6} \phi(x, y)}{\partial y^{6}}\right| \leq 72 /\left(x^{2}+y^{2}\right)^{2} .
$$


Expressions (3.5) and (3.10) show that, for each $j \in \mathcal{J}_{h}(\ell h, m h)$, the modulus of the contribution from the $\left(x_{j}, y_{j}\right)$ term of $s_{h}$ to the truncation error of the subtabulation formula $(2.2)$ when $f=s_{h}$ is at most the product

$$
6\left\{h /\left[\left(\ell h-x_{j}\right)^{2}+\left(m h-y_{j}\right)^{2}\right]^{1 / 2}\right\}^{6}\left|\lambda_{j}\left[\left(\ell h-x_{j}\right)^{2}+\left(m h-y_{j}\right)^{2}\right]\right|,
$$

where we have ignored the terms of magnitude $\mathcal{O}\left(h^{8}\right)$, but they are studied in the appendix. Thus formula (2.2) provides the relative accuracy

$$
6\left\{h /\left[\left(\ell h-x_{j}\right)^{2}+\left(m h-y_{j}\right)^{2}\right]^{1 / 2}\right\}^{6} /\left|\log \left[\left(\ell h-x_{j}\right)^{2}+\left(m h-y_{j}\right)^{2}\right]^{1 / 2}\right|
$$

in the $j$-th term of expression (3.3). We assume that the logarithm can also be ignored, this question being discussed in the next paragraph. Therefore, because we wish to work to a relative accuracy of $\epsilon$, we require the definition of $\mathcal{J}_{h}(\ell h, m h)$ to provide the bound

$$
\left[\left(\ell h-x_{j}\right)^{2}+\left(m h-y_{j}\right)^{2}\right]^{1 / 2} \geq(6 / \epsilon)^{1 / 6} h, \quad j \in \mathcal{J}_{h}(\ell h, m h) .
$$

It follows from condition (1.4) that it is suitable to set $\rho$ to the least integer that satisfies the inequality $\rho \geq(6 / \epsilon)^{1 / 6}$. For example, the values $\rho=14$ and $\rho=43$ are chosen in the cases $\epsilon=10^{-6}$ and $\epsilon=10^{-9}$ respectively. Therefore each application of the procedure of the second paragraph of this section requires $\mathcal{O}\left(n \epsilon^{-1 / 3}\right)$ operations, which causes the $\mathcal{O}\left(n \epsilon^{-1 / 3}|\log h|\right)$ component of the total work that is mentioned in the abstract.

Ignoring the logarithm of expression (3.12) is valid for certain scalings of the $x$ and $y$ variables. We have in mind that, from a practical point of view, it should not matter if the measurements in the pictures that are being compared are expressed in centimetres or in miles, for instance. At least one of these choices would admit our assumption, provided that a change in units does not damage the accuracy of formula (2.2). Now, if the $x$ and $y$ variables are scaled by a constant, then, instead of changing the log terms of expression (3.3), it is equivalent to add a certain quadratic polynomial to $s_{h}$. Further, we have chosen subtabulation formulae that are exact when they are applied to quadratic polynomials. It follows that the errors of the given approximations are independent of such changes of scale, so there is some reasonable justification for the use of inequality (3.13) when a relative accuracy of $\epsilon$ is required.

We complete this section by showing that the sixth order terms of the other formulae of Section 2 do not demand a larger value of $\rho$. We seek the sixth order Taylor series terms of the stencils of Figures 2 and 3 that are analogous to the sixth order term (2.9) of Figure 1. Therefore we continue to let $(\ell h, m h)$ be the origin in the expansion (2.4). Hence, writing the stencil of Figure 2 in the form

$$
\begin{aligned}
& f(\ell h, m h) \approx \sum_{ \pm}\left[\frac{39}{128} f(\ell h \pm h, m h)+\frac{39}{128} f(\ell h, m h \pm h)-\frac{1}{12} \overline{8} f(\ell h \pm 3 h, m h)\right. \\
& \left.-\frac{1}{12 \overline{8}} f(\ell h, m h \pm 3 h)\right]-\frac{3}{12} \overline{8} \sum_{ \pm, \pm}[f(\ell h \pm 2 h, m h \pm h)+f(\ell h \pm h, m h \pm 2 h)],
\end{aligned}
$$


we see that it has the sixth order terms

$$
\begin{aligned}
& \frac{h^{6}}{6 !}\left(\frac{\partial^{6} f(0,0)}{\partial x^{6}}+\frac{\partial^{6} f(0,0)}{\partial y^{6}}\right)\left[2 \times \frac{39}{128}-2 \times \frac{729}{128}-4 \times \frac{3}{128} \times(64+1)\right] \\
& -\frac{h^{4}}{4 !} \frac{h^{2}}{2 !}\left(\frac{\partial^{6} f(0,0)}{\partial x^{4} \partial y^{2}}+\frac{\partial^{6} f(0,0)}{\partial x^{2} \partial y^{4}}\right)\left[4 \times \frac{3}{128} \times(16+4)\right] \\
& =-\frac{3}{128} h^{6}\left(\frac{\partial^{6} f(0,0)}{\partial x^{6}}+\frac{\partial^{6} f(0,0)}{\partial y^{6}}\right)-\frac{5}{128} h^{6}\left(\frac{\partial^{6} f(0,0)}{\partial x^{4} \partial y^{2}}+\frac{\partial^{6} f(0,0)}{\partial x^{2} \partial y^{4}}\right) \\
& =-\frac{1}{96} h^{6}\left(\frac{\partial^{6} f(0,0)}{\partial x^{6}} \frac{\partial^{6} f(0,0)}{\partial y^{6}}\right),
\end{aligned}
$$

where the last line depends on the identity (2.8). The magnitude of this term is one eighth of expression (2.9), because the Euclidean diameter of the stencil of Figure 2 is the diameter of the stencil of Figure 1 divided by $2^{1 / 2}$. Moreover, because the stencil of Figure 3 denotes the functional

$$
\begin{gathered}
\sum_{ \pm}[9 f(\ell h \pm h, m h)-9 f(\ell h, m h \pm h)+f(\ell h \pm 3 h, m h)-f(\ell h, m h \pm 3 h)] \\
+\sum_{ \pm, \pm}[3 f(\ell h \pm h, m h \pm 2 h)-3 f(\ell h \pm 2 h, m h \pm h)]
\end{gathered}
$$

it has the sixth order terms

$$
\begin{aligned}
& \frac{h^{6}}{6 !}\left(\frac{\partial^{6} f(0,0)}{\partial x^{6}}-\frac{\partial^{6} f(0,0)}{\partial y^{6}}\right)[2 \times 9+2 \times 729+4 \times 3-4 \times 3 \times 64] \\
& \quad+\frac{h^{4}}{4 !} \frac{h^{2}}{2 !}\left(\frac{\partial^{6} f(0,0)}{\partial x^{4} \partial y^{2}}-\frac{\partial^{6} f(0,0)}{\partial x^{2} \partial y^{4}}\right)[4 \times 3 \times 4-4 \times 3 \times 16] \\
& =h^{6}\left(\frac{\partial^{6} f(0,0)}{\partial x^{6}}-3 \frac{\partial^{6} f(0,0)}{\partial x^{4} \partial y^{2}}+3 \frac{\partial^{6} f(0,0)}{\partial x^{2} \partial y^{4}}-\frac{\partial^{6} f(0,0)}{\partial y^{6}}\right) \\
& =4 h^{6}\left(\frac{\partial^{6} f(0,0)}{\partial x^{6}}-\frac{\partial^{6} f(0,0)}{\partial y^{6}}\right),
\end{aligned}
$$

where the last line depends on the elementary observation that equation (2.1) implies the identity

$$
\frac{\partial^{6} f(0,0)}{\partial x^{6}}+\frac{\partial^{6} f(0,0)}{\partial x^{4} \partial y^{2}}-\frac{\partial^{6} f(0,0)}{\partial x^{2} \partial y^{4}}-\frac{\partial^{6} f(0,0)}{\partial y^{6}}=0 .
$$

Remembering that the approximation (2.11) is formed by adding $1 / 128$ times the stencil of Figure 3 to the stencil of Figure 2, it follows from expressions (3.15) 
and (3.17) that the leading term of the error of formula (2.11) is the quantity

$$
-\frac{1}{96} h^{6}\left(\frac{\partial^{6} f(0,0)}{\partial x^{6}}+\frac{\partial^{6} f(0,0)}{\partial y^{6}}\right)+\frac{1}{32} h^{6}\left(\frac{\partial^{6} f(0,0)}{\partial x^{6}}-\frac{\partial^{6} f(0,0)}{\partial y^{6}}\right) \text {. }
$$

We also recall that we picked $\rho$ so that, if the bound $x^{2}+y^{2} \geq \rho^{2}$ holds and if $f(0,0)$ is replaced by $\phi(x, y)$ in expression (2.9), then inequality (3.10) makes the modulus of the sixth order term (2.9) acceptably small. In other words, due to the choice of $\rho$, the error term (2.9) is small enough because it satisfies the condition

$$
\left|\frac{1}{12} h^{6}\left(\frac{\partial^{6} \phi(x, y)}{\partial x^{6}}+\frac{\partial^{6} \phi(x, y)}{\partial y^{6}}\right)\right| \leq 6 h^{6} /\left(x^{2}+y^{2}\right)^{2} .
$$

Therefore the choice of $\rho$ also ensures that expression (3.19) is suitably small if the definition (3.6) implies the inequality

$$
\begin{gathered}
\left|-\frac{1}{96} h^{6}\left(\frac{\partial^{6} \phi(x, y)}{\partial x^{6}}+\frac{\partial^{6} \phi(x, y)}{\partial y^{6}}\right)+\frac{1}{32} h^{6}\left(\frac{\partial^{6} \phi(x, y)}{\partial x^{6}}-\frac{\partial^{6} \phi(x, y)}{\partial y^{6}}\right)\right| \\
\leq 6 h^{6} /\left(x^{2}+y^{2}\right)^{2}
\end{gathered}
$$

Now the last of the equations (3.7) and symmetry give the identity

$$
\frac{\partial^{6} \phi(x, y)}{\partial x^{6}}-\frac{\partial^{6} \phi(x, y)}{\partial y^{6}}=\left(48 x^{6}-720 x^{4} y^{2}+720 x^{2} y^{4}-48 y^{6}\right) /\left(x^{2}+y^{2}\right)^{5}
$$

so, because the numerator of the right hand side can be expressed in the forms

$$
48\left(x^{2}+y^{2}\right)^{3}-96 y^{2}\left(3 x^{2}-y^{2}\right)^{2} \text { and } 96 x^{2}\left(3 y^{2}-x^{2}\right)^{2}-48\left(x^{2}+y^{2}\right)^{3}
$$

we have the bound

$$
\left|\frac{\partial^{6} \phi(x, y)}{\partial x^{6}}-\frac{\partial^{6} \phi(x, y)}{\partial y^{6}}\right| \leq 48 /\left(x^{2}+y^{2}\right)^{2}
$$

Conditions (3.20) and (3.24) show that the required inequality (3.21) is satisfied even if we reduce the constant on the right hand side from 6 to $9 / 4$.

We have not yet, however, allowed for the important point that the function values $f(\ell h \pm h, m h)$ of the estimate (2.11) include sixth order errors, because the procedure of Section 2 calculates them by applying formula (2.2). Therefore, in view of the derivation of expression (2.9), we should add the term

$$
2 \times \frac{3}{8} \times \frac{1}{12} h^{6}\left(\frac{\left.\partial^{6} f(0,0), \frac{\partial^{6} f(0,0)}{\partial x^{6}}\right)}{\partial y^{6}}\right)
$$


to the quantity (3.19). Thus, after replacing $f(\ell h \pm h, m h)$ by their approximations, the estimate (2.11) has the leading error term

$$
\frac{5}{96} h^{6}\left(\frac{\partial^{6} f(0,0)}{\partial x^{6}}+\frac{\partial^{6} f(0,0)}{\partial y^{6}}\right)+\frac{1}{32} h^{6}\left(\frac{\partial^{6} f(0,0)}{\partial x^{6}}-\frac{\partial^{6} f(0,0)}{\partial y^{6}}\right),
$$

which is acceptably small, because conditions (3.20) and (3.24) give the bound

$$
\begin{gathered}
\left|\frac{5}{96} h^{6}\left(\frac{\partial^{6} \phi(x, y)}{\partial x^{6}}+\frac{\partial^{6} \phi(x, y)}{\partial y^{6}}\right)+\frac{1}{32} h^{6}\left(\frac{\partial^{6} \phi(x, y)}{\partial x^{6}}-\frac{\partial^{6} \phi(x, y)}{\partial y^{6}}\right)\right| \\
\leq \frac{21}{4} h^{6} /\left(x^{2}+y^{2}\right)^{2}
\end{gathered}
$$

Similarly, the choice of $\rho$ also ensures that the accuracy of formula (2.12) is adequate.

We have not investigated analytically whether a sequence of applications of the method of Section 2 can cause an unacceptably large accumulation of errors. In particular, the stability properties of the stencils when they are used recursively have not been considered, but similar questions are studied in the analysis of algorithms for "subdivision" (see Dyn, 1992, for example). The coefficients of our subtabulation formulae seem to be harmless, and no difficulties have occurred in numerical computations. Further, if the function value $f(\ell h, m h)$ is given to the procedure of Section 2, and if it includes a relatively large error due to the singularity of the thin plate spline at the interpolation point $\left(x_{j}, y_{j}\right)$, then $(\ell h, m h)$ must be close to the boundary of the neighbourhood $\mathcal{N}_{j}(\kappa h)$, for some integer $\kappa$ that is a power of 2 satisfying $\kappa \geq 2$. Therefore, assuming $\rho \geq 10$ for instance, we have the advantage that, for the current and future values of $h$, the function value $f(\ell h, m h)$ will feature only in subtabulation formulae that have relatively small new errors arising from the singularity at $\left(x_{j}, y_{j}\right)$.

\section{Numerical results and discussion}

The Fortran software has solved many calculations. It was developed by the author in 1990, and since then has been made to run faster at Barrodale Computing Services. Some typical examples of execution times of the original version are shown in Table 1. The given figures are computation times in seconds on a Sparc workstation for a range of grid sizes and values of $n$ with $\epsilon=10^{-6}$, but they include some inconsistencies of at least $1 \%$ due to their dependence on the scheduling of a Unix operating system. Nevertheless, the table distinguishes the two main components of the total work, which are the use of the subtabulation formulae and the corrections that allow for the discontinuities in $s_{h}$, these components being a small multiple of $h^{-2}$ and a large multiple of $n|\log h|$ respectively, where $h$ is the final mesh size and $n$ is the number of interpolation points. Indeed, because the 


\begin{tabular}{|c||r|r|r|r|r|}
\hline Grid size & $n=25$ & $n=50$ & $n=100$ & $n=200$ & $n=400$ \\
\hline $100 \times 100$ & 0.85 & 1.64 & 3.20 & 6.30 & 12.44 \\
$200 \times 200$ & 1.36 & 2.41 & 4.43 & 8.66 & 16.74 \\
$400 \times 400$ & 2.54 & 3.77 & 6.23 & 11.66 & 21.08 \\
$800 \times 800$ & 6.59 & 7.94 & 10.78 & 16.49 & 27.96 \\
\hline
\end{tabular}

Table 1: Some timings in seconds

entries in the first two rows of the table tend to be proportional to $n$, we deduce that the work of the correction procedure requires about $n / 100$ seconds whenever the mesh size is halved. Further, the $n=25$ column provides upper bounds on the total times that are taken by the subtabulation formulae. On the other hand, if all the values of $s$ on the final $800 \times 800$ grid are calculated separately from the definition (1.2), then 395.56 seconds are required when $n=25$, the time when $n=400$ being about 16 times longer. Thus it is clear that the gains that are provided by the given algorithm are very substantial.

Much finer meshes can be treated efficiently, even when there is not enough computer storage to hold the final grid of function values. The reason is that one can divide the final grid into pieces that can be accommodated in storage and one can apply the algorithm to each piece separately. Of course the overlap between pieces that is suggested by Figure 5 is needed, and it will happen that some of the interpolation points $\left\{\left(x_{j}, y_{j}\right): j=1,2, \ldots, n\right\}$ will lie outside the region that is covered by the grid of a typical subcalculation. These features do not introduce any difficulties.

There are some subtabulation formulae that provide $\mathcal{O}\left(h^{6}\right)$ accuracy and that require less work than the stencils of Figures 1 and 4 . In particular, one could apply the estimate

$$
f(\ell h, m h) \approx \frac{1}{25 \overline{6}} \sum_{ \pm}[150 f(\ell h \pm h, m h)-25 f(\ell h \pm 3 h, m h)+3 f(\ell h \pm 5 h, m h)]
$$

along grid lines that are parallel to the $x$-axis, and of course there is an analogous formula in the $y$-direction. Perhaps the only objection to this method is that it does not have the two-dimensional structure that is inherent in the main calculation. Further, the idea of using linear formulae of the type (4.1) near the edges of the regions $\left\{\mathcal{N}_{j}(h): j=1,2, \ldots, n\right\}$ could avoid many of the time-consuming discontinuity corrections of the current algorithm. Corrections would be needed near the corners of the square neighbourhoods, however, and also where the boundaries of two different neighbourhoods intersect at right angles.

Alternatively, one can avoid the discontinuities altogether by working with values of the original function $s$ throughout the calculation. In this case one would have to correct the values that are given by the subtabulation formulae 
within the neighbourhoods $\left\{\mathcal{N}_{j}(h): j=1,2, \ldots, n\right\}$ after each cycle through the operations of Section 2. Thus the work of the modifications would be proportional to $n \rho^{2}$, instead of the present much larger multiple of $n \rho$ plus a smaller multiple of $n \rho^{2}$. In view of the entries in Table 1 , it is probable that, for typical values of $\rho$, it would be more efficient to prefer $f=s$ instead of $f=s_{h}$.

In general, radial basis function methods are expensive in comparison with approximation techniques that use piecewise polynomials, because the work of calculating a value $s(x)$ of a radial basis function interpolant is proportional to the number of interpolation points. Therefore it is important that we have shown that substantial savings can be made when $s(x)$ is required for many different values of $x$ on a regular grid. Further, techniques have been developed for the case when the points $x$ are in general position, such as the fast multipole method of Greengard and Rokhlin (1987), which treats clusters of interpolation points that are sufficiently remote from the current $x$ as single Laurent series. The application of this method to thin plate splines is described and analysed by Beatson and Newsam (1992). Such developments are increasing greatly the usefulness of radial basis function methods for the solution of a wide range of multivariate approximation problems.

\section{Appendix: The truncation errors of the subtabulation formulae}

When the given algorithm applies the subtabulation formula (2.2), the resultant truncation error is expression (3.5). Further, in view of inequality (3.20) and the definition (3.2), we have the bound

$$
\begin{aligned}
\left|\frac{1}{12} h^{6} \lambda_{j}\left(\frac{\partial^{6} \phi\left(\ell h-x_{j}, m h-y_{j}\right)}{\partial x^{6}}+\frac{\partial^{6} \phi\left(\ell h-x_{j}, m h-y_{j}\right)}{\partial y^{6}}\right)\right| \\
\leq 6 h^{6}\left|\lambda_{j}\right| /\left[\left(\ell h-x_{j}\right)^{2}+\left(m h-y_{j}\right)^{2}\right]^{2} \\
\leq 6 \rho^{-6}\left|\lambda_{j}\right|\left[\left(\ell h-x_{j}\right)^{2}+\left(m h-y_{j}\right)^{2}\right], \quad j \in \mathcal{J}_{h}(\ell h, m h) .
\end{aligned}
$$

Usually, therefore, we expect our choice of $\rho$, namely the least integer that satisfies $\rho \geq(6 / \epsilon)^{1 / 6}$, to provide the required relative accuracy of $\epsilon$. The main purpose of this appendix is to investigate whether the higher order terms of expression (3.5) can cause serious damage to this expectation.

We derive the total contribution to the truncation error (3.5) from a single value of $j$. We assume without loss of generality that $\lambda_{j}=1$ and that $\left(x_{j}, y_{j}\right)$ is the origin. Therefore we consider the difference between the two sides of formula (2.2) when we have $f \equiv \phi$ and $\max [|\ell|,|m|] \geq \rho$. Since the choice of $\rho$ is guided by inequality (3.20), we wish to show that the modulus of this total contribution is not much larger than $6 h^{6} /\left[(\ell h)^{2}+(m h)^{2}\right]^{2}=6 h^{2} /\left(\ell^{2}+m^{2}\right)^{2}$.

We are going to employ the Taylor series expansion of $\phi$ about the point 
$(x, y)=(\ell h, m h)$. Fortunately the following procedure yields all the required derivatives of $\phi$. We write the third equation of expression (3.7) in the form

$$
\frac{\partial^{3} \phi(x, y)}{\partial x^{3}}-\frac{x+2 i y}{(x+i y)^{2}}+\frac{x-2 i y}{(x-i y)^{2}},
$$

where $i=(-1)^{1 / 2}$. Then the Leibniz formula gives the higher order derivatives

$$
\frac{\partial^{j} \phi(x, y)}{\partial x^{j}}=(-1)^{j+1}(j-3) !\left(\frac{x+(j-1) i y}{(x+i y)^{j-1}}, \frac{x-(j-1) i y}{(x-i y)^{j-1}}\right), \quad j \geq 3 .
$$

Further, the $k$-th derivative of this expression with respect to $y$ has the value

$$
(-1)^{j}(j+k-3) !\left((-i)^{k} \frac{(k-1) x-(j-1) i y}{(x+i y)^{j+k-1}}+i^{k} \frac{(k-1) x+(j-1) i y}{(x-i y)^{j+k-1}}\right) .
$$

It is straightforward to verify that this formula is also valid for $0 \leq j \leq 2$ provided that we have $j+k \geq 3$. We state this conclusion formally.

Lemma 1. The thin plate spline function (3.6) has the derivative

$$
\frac{\partial^{j+k} \phi(x, y)}{\partial x^{j} \partial y^{k}}=2(-1)^{j}(j+k-3) ! \Re\left(i^{k} \frac{(k-1) x+(j-1) i y}{(x-i y)^{j+k-1}}\right),
$$

where $j$ and $k$ are any nonnegative integers such that $j+k \geq 3$, and where $\Re$ denotes the real part of the term in the large brackets.

Because of the smoothness properties of $\phi$, it can be proved that the Taylor series expansion

$$
\phi(x+\xi, y+\eta)=\sum_{j=0}^{\infty} \sum_{k=0}^{\infty} \frac{\xi^{j}}{j !} \frac{\eta^{k}}{k !} \frac{\partial^{j+k} \phi(x, y)}{\partial x^{j} \partial y^{k}}
$$

is valid if the double sum is absolutely convergent. We will deduce from the following lemma that this property is enjoyed by all the values of $x, y, \xi$ and $\eta$ that we allow in the subtabulation formulae of Section 2.

Lemma 2. The sum (A.6) is absolutely convergent if its variables satisfy the inequality

$$
|\xi|+|\eta|<\left(x^{2}+y^{2}\right)^{1 / 2}
$$

Proof: Equation (A.5) implies the bound

$$
\left|\frac{\partial^{j+k} \phi(x, y)}{\partial x^{j} \partial y^{k}}\right| \leq \frac{2(j+k-3) ! \max [k-1, j-1]}{\left(x^{2}+y^{2}\right)^{(j+k-2) / 2}} \frac{(j+k) !}{\left(x^{2}+y^{2}\right)^{(j+k-2) / 2}}
$$


when $j+k \geq 3$. Moreover, condition (A.7) implies that the sum

$$
\sum_{t=0}^{\infty}\left(\frac{|\xi|+|\eta|}{\left(x^{2}+y^{2}\right)^{1 / 2}}\right)^{t}=\sum_{j=0}^{\infty} \sum_{k=0}^{\infty} \frac{(j+k) !}{j ! k !} \frac{|\xi|^{j}|\eta|^{k}}{\left(x^{2}+y^{2}\right)^{(j+k) / 2}}
$$

is finite. Therefore the relation

$$
\sum_{\substack{j=0 \\ j+k \geq 3}}^{\infty} \sum_{k=0}^{\infty}\left|\frac{\xi^{j}}{j !} \frac{\eta^{k}}{k !} \frac{\partial^{j+k} \phi(x, y)}{\partial x^{j} \partial y^{k}}\right|<\left(x^{2}+y^{2}\right) \sum_{t=0}^{\infty}\left(\frac{|\xi|+|\eta|}{\left(x^{2}+y^{2}\right)^{1 / 2}}\right)^{t}
$$

holds, which shows that the lemma is true.

We assume that the parameter $\rho$ of the algorithm satisfies $\rho \geq 10$. Hence, because we have the conditions

$$
(x, y)=(\ell h, m h), \quad\left(x_{j}, y_{j}\right)=(0,0) \quad \text { and } \quad j \in \mathcal{J}_{h}(\ell h, m h),
$$

the right hand side of inequality (A.7) is bounded below by $10 h$. Moreover, the values of $(\xi, \eta)$ that will occur in equation (A.6) are the displacements from the centres to the data points of the stencils of Figures 1-4, so they satisfy the condition $|\xi|+|\eta| \leq 6 h$. It follows that Lemma 2 is applicable. Therefore the Taylor series expansion (A.6) gives the formula

$$
\phi(x+\xi, y+\eta)=\sum_{t=0}^{\infty}\left(\sum_{\substack{j, k \\ j+k=t}} \frac{\xi^{j}}{j !} \frac{\eta^{k}}{k !} \frac{\partial^{j+k} \phi(x, y)}{\partial x^{j} \partial y^{k}}\right)=\sum_{t=0}^{\infty} \psi_{t}(\xi, \eta)
$$

say. Further, we find next that the sum inside the large brackets can be calculated analytically for all $t \geq 3$.

Specifically, equation (A.5) implies the value

$$
\begin{aligned}
& \psi_{t}(\xi, \eta)=2(t-3) ! \Re\left(\sum_{\substack{j, k \\
j+k=t}} \frac{(-\xi)^{j}(i \eta)^{k}(k-1) x+(j-1) i y}{j ! k !(x-i y)^{t-1}}\right) \\
& =\frac{2(t-3) !}{t !} \Re\left(\frac{(-x-i y)(-\xi+i \eta)^{t}+t(i x \eta-i y \xi)(-\xi+i \eta)^{t-1}}{(x-i y)^{t-1}}\right)
\end{aligned}
$$

where the last line is derived from the binomial expansions of $(-\xi+i \eta)^{t}$ and $(-\xi+i \eta)^{t-1}$. In order to simplify this expression in a way that employs the rotational symmetry of the thin plate spline function $\phi$, we introduce the notation

$$
d=\left(x^{2}+y^{2}\right)^{1 / 2}, \quad \delta=\left(\xi^{2}+\eta^{2}\right)^{1 / 2} \quad \text { and } \quad \theta=\arg (\xi+i \eta)-\arg (x+i y)
$$


so we have the elementary relations

$$
x \xi+y \eta=d \delta \cos \theta \quad \text { and } \quad x \eta-y \xi=d \delta \sin \theta .
$$

It follows that we can write expression (A.13) in the form

$$
\begin{aligned}
& 2\left(\delta^{t} / d^{t-2}\right) \Re\left(-(-\cos \theta+i \sin \theta)^{t}+i t \sin \theta(-\cos \theta+i \sin \theta)^{t-1}\right) /[t(t-1)(t-2)] \\
& \quad=(-1)^{t}\left(\delta^{t} / d^{t-2}\right)[(t-2) \cos (t \theta)-t \cos (t \theta-2 \theta)] /[t(t-1)(t-2)], \quad \text { (A.16) }
\end{aligned}
$$

which gives the following assertion.

Lemma 3. Let $\phi$ be the thin plate spline function (3.6) and let the real variables $x, y, \xi$ and $\eta$ satisfy inequality (A.7). Then, for every integer $t$ such that $t \geq 3$, the sum in the large brackets of the Taylor series expansion (A.12) has the value

$$
\psi_{t}(\xi, \eta)=(-1)^{t} \frac{[(t-2) \cos (t \theta)-t \cos (t \theta-2 \theta)]\left(\xi^{2}+\eta^{2}\right)^{t / 2}}{t(t-1)(t-2)\left(x^{2}+y^{2}\right)^{(t-2) / 2}}
$$

where $\theta$ is specified in the definition (A.14).

We apply this lemma to the stencil of Figure 1, so we require the Taylor series expansion about $(x, y)=(\ell h, m h)$ of every function value on the right hand side of formula (2.2). We let $\theta_{0}, \theta_{1}$ and $\theta_{2}$ be the values of $\theta$ in the definition (A.14) when $(\xi, \eta)$ is $(3 h, h),(h, h)$ and $(h, 3 h)$ respectively. Therefore we have the relations

$$
\cos \left(\theta_{1}-\theta_{0}\right)=\cos \left(\theta_{2}-\theta_{1}\right)=2 / 5^{1 / 2}
$$

In view of the symmetry of Figure 1 , the $\cos (t \theta)$ term of expression (A.17) causes the data values with the weights $-3 / 128$ to be multiplied by the factor

$$
\begin{aligned}
\sum_{j=0}^{3}\left[\cos \left(t \theta_{0}+\frac{1}{2} j t \pi\right)+\cos \left(t \theta_{2}+\frac{1}{2} j t \pi\right)\right] & =4 \delta_{t}\left[\cos \left(t \theta_{0}\right)+\cos \left(t \theta_{2}\right)\right] \\
& =8 \delta_{t} \cos \left(t \theta_{1}\right) \cos \left[t\left(\theta_{1}-\theta_{0}\right)\right]
\end{aligned}
$$

where elementary properties of the cosine function imply that $\delta_{t}$ has the value $\delta_{t}=1$ if the integer $t$ is a multiple of 4 , but otherwise $\delta_{t}=0$. The corresponding factor for the other weights of the Figure 1 stencil is $4 \delta_{t} \cos \left(t \theta_{1}\right)$, and of course the factors of the $\cos (t \theta-2 \theta)$ part of expression (A.17) are obtained by reducing $t$ by 2 . Thus we deduce the following result from Lemma 3.

Lemma 4. Let $\phi$ be the thin plate spline function (3.6), let the integers $\ell$ and $m$ satisfy $\max [|\ell|,|m|] \geq \rho \geq 10$, let $\theta$ and $\hat{\theta}$ be the angles $\frac{1}{4} \pi-\arg (\ell+i m)$ and $\cos ^{-1}\left(2 / 5^{1 / 2}\right)$ respectively, and let $t$ be any integer such that $t \geq 3$. Then the $t$-th 
order term of the error of the approximation (2.2) is zero if $t$ is odd, it has the value

$$
4 h^{2}\left[\frac{39}{128} 2^{t / 2}-\frac{3}{64} 10^{t / 2} \cos (t \hat{\theta})-\frac{1}{128} 18^{t / 2}\right] \cos (t \theta) /\left[t(t-1)\left(\ell^{2}+m^{2}\right)^{(t-2) / 2}\right]
$$

if $t$ is divisible by 4 , and it has the value

$4 h^{2}\left[-\frac{39}{128} 2^{t / 2}+\frac{3}{64} 10^{t / 2} \cos (t \hat{\theta}-2 \hat{\theta})+\frac{1}{128} 18^{t / 2}\right] \cos (t \theta-2 \theta) /\left[(t-1)(t-2)\left(\ell^{2}+m^{2}\right)^{(t-2) / 2}\right]$

if $t$ is twice an odd integer.

It follows from this lemma that, when $t=4,6,8$ and 10 , the $t$-th order truncation errors of formula (2.2) are the expressions

$$
0, \frac{6 h^{2} \cos (4 \theta)}{\left(\ell^{2}+m^{2}\right)^{2}}, \frac{-30 h^{2} \cos (8 \theta)}{\left(\ell^{2}+m^{2}\right)^{3}} \text { and } \frac{600 h^{2} \cos (8 \theta)}{\left(\ell^{2}+m^{2}\right)^{4}}
$$

respectively. Remembering the condition $\max [|\ell|,|m|] \geq 10$, we deduce that the third and fourth of these expressions are at most $5 \%$ and $1 \%$ of the bound $6 h^{2} /\left(\ell^{2}+\right.$ $\left.m^{2}\right)^{2}$ on the sixth order truncation error, given at the end of the second paragraph of the appendix and verified by the second of the expressions (A.22). The lemma also implies that the modulus of the total contribution to the truncation error from all values of $t$ that satisfy $t \geq 12$ is less than the number

$$
\begin{aligned}
\sum_{k=6}^{\infty} h^{2}\left[\frac{39}{32} 2^{k}+\frac{3}{16} 10^{k}+\frac{1}{32} 18^{k}\right] /\left[132\left(\ell^{2}+m^{2}\right)^{k-1}\right] \\
=\left[6 h^{2} /\left(\ell^{2}+m^{2}\right)^{2}\right] \sum_{k=6}^{\infty}\left[\frac{13}{1056} 2^{k-3}+\frac{125}{528} 10^{k-3}+\frac{81}{352} 18^{k-3}\right] /\left(\ell^{2}+m^{2}\right)^{k-3} \\
\leq\left[6 h^{2} /\left(\ell^{2}+m^{2}\right)^{2}\right]\left[\frac{13}{1056}(0.02)^{3} / 0.98+\frac{125}{528}(0.1)^{3} / 0.9+\frac{81}{352}(0.18)^{3} / 0.82\right] \\
<0.002\left[6 h^{2} /\left(\ell^{2}+m^{2}\right)^{2}\right] .
\end{aligned}
$$

Therefore, when $\rho \geq 10$, the total truncation error of formula (2.2) does not exceed the sixth order bound of Section 3 by more than $6.2 \%$, so the given algorithm provides good accuracy in its calculation of $s(\ell h, m h)$, when $\ell$ and $m$ are both odd.

Of course there are analogues of Lemma 4 for the stencils of Figures 2 and 3. Indeed, the modifications to Lemma 4 that make it relevant to Figure 2 are as follows. We allow for the change in orientation of the stencil by altering the definition of $\theta$ to $-\arg (\ell+i m)$, and we allow for the change in scale by multiplying expressions (A.20) and (A.21) by the factor $2^{-t / 2}$. It follows that the higher order errors of the Figure 2 stencil are negligible. Therefore, because formulae (2.11) and (2.12) are derived by adding and subtracting 1/128 times the Figure 3 functional 
to and from the Figure 2 stencil, it remains to show that, when $\rho \geq 10$, the modulus of this functional is substantially less than the quantity $128 \times 6 h^{2} /\left(\ell^{2}+m^{2}\right)^{2}$. We employ the remark that, in view of the symmetry and antisymmetry properties of Figure 3, the argument that yielded Lemma 4 now provides the following assertion.

Lemma 5. Let $\phi$ be the thin plate spline function (3.6), let the integers $\ell$ and $m$ satisfy $\max [|\ell|,|m|] \geq \rho \geq 10$, let $\theta$ and $\hat{\theta}$ be the angles $-\arg (\ell+i m)$ and $\cos ^{-1}\left(2 / 5^{1 / 2}\right)$ respectively, and let $t$ be any integer such that $t \geq 3$. Then the $t$-th order term of the functional of Figure 3 is zero if $t$ is odd, it has the value

$$
4 h^{2}\left[-9+6 \times 5^{t / 2} \cos (t \hat{\theta}-2 \hat{\theta})-3^{t}\right] \cos (t \theta-2 \theta) /\left[(t-1)(t-2)\left(\ell^{2}+m^{2}\right)^{(t-2) / 2}\right]
$$

if $t$ is divisible by 4 , and it has the value

$$
4 h^{2}\left[9-6 \times 5^{t / 2} \cos (t \hat{\theta})+3^{t}\right] \cos (t \theta) /\left[t(t-1)\left(\ell^{2}+m^{2}\right)^{(t-2) / 2}\right]
$$

if $t$ is twice an odd integer.

Therefore, when $t=4,6,8$ and 10 , the $t$-th order terms of the functional that is shown in Figure 3 are the expressions

$$
0, \frac{192 h^{2} \cos (6 \theta)}{\left(\ell^{2}+m^{2}\right)^{2}}, \frac{-960 h^{2} \cos (6 \theta)}{\left(\ell^{2}+m^{2}\right)^{3}} \text { and } \frac{2688 h^{2} \cos (10 \theta)}{\left(\ell^{2}+m^{2}\right)^{4}} \text {. }
$$

respectively. The first two expressions are expected from the analysis of Section 3, in particular inequalities (3.17) and (3.24) imply the attainable bound $192 h^{2} /\left(\ell^{2}+m^{2}\right)^{2}$ on the modulus of the sixth order term. Further, we see that the moduli of the eighth and tenth order terms are substantially less than 128 times the greatest moduli of the corresponding terms of expression (A.22). Further, a comparison of Lemmas 4 and 5 shows that this property is also enjoyed by all larger values of $t$. Hence the higher order terms of the Figure 3 stencil induce errors in the subtabulation formulae (2.11) and (2.12) that are substantially less than the corresponding errors of formula (2.2). We conclude from the analysis of this appendix that, when typical or high accuracy is required from the subtabulation algorithm, then it is adequate to determine $\rho$ in the given way that depends on sixth order error estimates.

Acknowledgements: I am very grateful to Ian Barrodale for suggesting to me that it should be possible to develop an algorithm for tabulating a thin plate spline on a fine square grid that is much faster than direct evaluation. I have enjoyed developing the given procedure and contributing to its successful use by Barrodale Computing Services on a wide range of applications. I also offer my thanks to Rick Beatson and Martin Buhmann for their valuable comments on the first draft of this paper. 


\section{References}

I. Barrodale, M. Berkley and D. Skea (1992), "Warping digital images using thin plate splines", presented at the Sixth Texas International Symposium on Approximation Theory (Austin, January, 1992).

R.K. Beatson and G.N. Newsam (1992), "Fast evaluation of radial basis functions: I", preprint (to be published in Comp. Maths. Appls.).

J. Duchon (1977), "Splines minimizing rotation-invariant seminorms in Sobolev spaces", in Constructive Theory of Functions of Several Variables, Lecture Notes in Mathematics 571, eds. W. Schempp and K. Zeller, Springer-Verlag (Berlin), pp. 85-100.

N. Dyn (1992), "Subdivision schemes in computer-aided geometric design", in Advances in Numerical Analysis II: Wavelets, Subdivision Algorithms and Radial Functions, ed. W.A. Light, Oxford University Press (Oxford) (to be published).

L. Greengard and V. Rokhlin (1987), "A fast algorithm for particle simulations", J. Comp. Phys., Vol. 73, pp. 325-348. 